\title{
Engineering particle trajectories in microfluidic flows using speckle light fields
}

Giorgio Volpe

Giovanni Volpe

Sylvain Gigan 


\title{
Engineering Particle Trajectories in Microfluidic Flows Using Speckle Light Fields
}

\author{
Giorgio Volpe ${ }^{* a}$, Giovanni Volpe ${ }^{\mathrm{b}, \mathrm{c}}$, Sylvain Gigan ${ }^{\mathrm{a}}$ \\ ${ }^{a}$ Laboratoire Kastler Brossel, UMR8552 of CNRS and Université Pierre et Marie Curie, Ecole \\ Normale Supérieure and Collège de France, 24 rue Lhomond, 75005 Paris, France; \\ ${ }^{\mathrm{b}}$ Physics Department, Bilkent University, Cankaya, 06800 Ankara, Turkey; \\ 'UNAM - National Nanotechnology Research Center, Bilkent University, Cankaya, 06800 Ankara, \\ Turkey
}

\begin{abstract}
Optical tweezers have been widely used in physics, chemistry and biology to manipulate and trap microscopic and nanoscopic objects. Current optical trapping techniques rely on carefully engineered setups to manipulate nanoscopic and microscopic objects at the focus of a laser beam. Since the quality of the trapping is strongly dependent on the focus quality, these systems have to be very carefully aligned and optimized, thus limiting their practical applicability in complex environments. One major challenge for current optical manipulation techniques is the light scattering occurring in optically complex media, such as biological tissues, turbid liquids and rough surfaces, which give rise to apparently random light fields known as speckles. Here, we discuss an experimental implementation to perform optical manipulation based on speckles. In particular, we show how to take advantage of the statistical properties of speckle patterns in order to realize a setup based on a multimode optical fiber to perform basic optical manipulation tasks such as trapping, guiding and sorting. We anticipate that the simplicity of these "speckle optical tweezers" will greatly broaden the perspectives of optical manipulation for real-life applications.
\end{abstract}

Keywords: optical forces, Brownian motion, speckles, optical tweezers, light scattering, optical manipulation, random light fields

\section{INTRODUCTION}

Since their introduction in the 1970s [1,2], optical tweezers have been widely applied to non-invasively manipulate micro- and nano-objects, such as cells, organelles and macromolecules [3-5]. They have, therefore, gained increasing importance as tools in microbiology and biophysics both for fundamental studies [6] and for more advanced applications such as optical sorting and optical delivery [3,7-8]. In particular, the development of techniques based on reconfigurable spatially extended patterns of light, such as multiple traps [3,9-12] or periodic potentials [13-17], offers the promise of high throughput optical methods to be applied both in static and moving fluids. Also, particles' delivery, trapping and manipulation over extended areas was demonstrated near a surface employing the evanescent fields associated, for example, to surface plasmons [18] or to optical waveguides [19].

Most of current optical manipulation techniques rely either on carefully engineered optical systems or advanced fabrication tools. Although such conditions can be routinely met in research laboratories, similar requirements, sometimes very stringent, limit the applicability of these techniques, e.g., to biomedical and microfluidic applications, where simplicity, low-cost and high-throughput are paramount. Moreover, one major challenge common to all these techniques is the light scattering occurring in optically complex media, such as biological tissues, turbid liquids and rough surfaces, which naturally gives rise to apparently random light fields known as speckles [20]. Earlier experimental works showed trapping of atoms and particles in a gas by high-intensity speckle light fields [21-24], while both static and time-varying speckle fields were related to the emergence of anomalous diffusion in colloids [25-29]. Recently, we derived a theory to describe the motion of a Brownian particle in a speckle light field which allowed us to demonstrate numerically how a speckle field can be used to control the motion a Brownian particle in the limit of particles much smaller than the light wavelength (dipole approximation) [29]. However, apart from these previous studies, the intrinsic

"'giorgio.volpe@lkb.ens.fr

Optical Trapping and Optical Micromanipulation XI, edited by Kishan Dholakia, Gabriel C. Spalding, Proc. of SPIE Vol. 9164, 91640I - (c) 2014 SPIE CCC code: $0277-786 \mathrm{X} / 14 / \$ 18 \cdot$ doi: $10.1117 / 12.2061040$ 
randomness of speckle patterns is largely considered a nuisance to be minimized for most purposes in optical manipulation [30-31]. In fact, similar and even more complex effects have been extensively studied using periodic potentials rather than random potentials: these studies include the demonstration of guiding and sorting particles using either moving periodic potentials [16-17], static periodic potentials in microfluidic flows [7,11-12], or optical ratchets based on spatially symmetric energy landscapes [32-33]. Only recently we were able to demonstrate experimentally a novel technique for the collective manipulation of micrometer-sized particles in microfluidic flows based on extended static and time-varying speckle light field [34].

Here, we discuss an experimental implementation to perform optical manipulation based on speckles. In particular, we show how to take advantage of the statistical properties of speckle patterns in order to realize a setup based on a multimode optical fiber to perform basic optical manipulation tasks such as trapping, guiding and sorting. We anticipate that the simplicity of these "speckle optical tweezers" will greatly broaden the perspectives of optical manipulation for real-life applications.

\section{EXPERIMENTAL IMPLEMENTATION}

The speckle optical tweezers setup is schematically depicted in Figure 1(a). Aqueous dispersions of colloidal spheres are driven by a syringe pump with adjustable infusion flow rate (Harvard Apparatus Pump 11 Elite) through a microfluidic channel. The speckle light pattern for their optical manipulation (Figure 1(b)) is generated by coupling a laser beam (Coherent Verdi, maximum power $5 \mathrm{~W}, \lambda=532 \mathrm{~nm}$ ) into a multimode optical fiber (core diameter $105 \mu \mathrm{m}, \mathrm{NA}=0.22$ ). The random appearance of speckle light patterns is the result of the interference of a large number of optical waves with random phases, corresponding to different eigenmodes of the fiber. More generally, speckle patterns can be generated by different processes: scattering of a laser on a rough surface, multiple scattering in an optically complex medium, or, like in this work, mode-mixing in a multimode fiber. The method chosen in this work provides some practical advantages over other methods, namely the generation of homogeneous speckle fields over controllable areas, flexibility and portability in the implementation of the device, as well as higher transmission efficiency. In our setup, the fiber output is brought in close proximity of the upper wall of the microfluidic channel by a micrometric two-axis mechanical stage that also guarantees the possibility of translating the speckle in the direction perpendicular to the fluid flow. Optical scattering forces push the particles in the direction of light propagation towards the lower wall of the microfluidic channel, so that they effectively confine the particles in a quasi two-dimensional space [1]. The particles are then tracked by digital video microscopy [35] on a color CMOS camera. The incoherent illumination for the tracking is provided by a LED at $\lambda=625 \mathrm{~nm}$ coupled into the same fiber using a dichroic mirror. Figure 1(c) shows the normalized spatial autocorrelation function of a typical speckle pattern interacting with the particles (Figure 1(b)), whose full width half maximum $(\mathrm{FWHM}=2.20 \pm 0.24 \mu \mathrm{m})$ provides an estimation of the average speckle grain size (Figure 1(d)), as defined by the diffraction process that generates the speckle pattern itself [20].
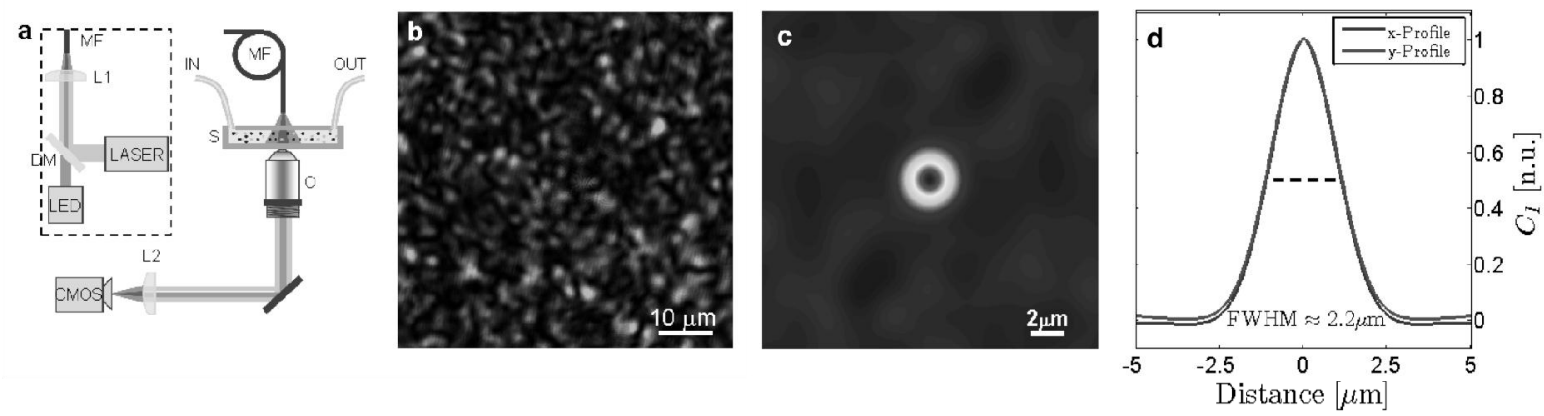

Figure 1. Speckle optical tweezers setup. (a) Schematic of the speckle optical tweezers setup. A laser beam $(\lambda=532 \mathrm{~nm})$ and incoherent light from an LED $(\lambda=625 \mathrm{~nm})$ are coupled into a multimode optical fiber $(105-\mu \mathrm{m}$ core, NA $=0.22)$ making use of a dichroic mirror (DM) and a lens (L1). The fiber delivers the light to a microfluidic channel (S) where aqueous dispersions of particles are flowed by a syringe pusher. The fiber output is mounted on a two-axis mechanical stage, which guarantees the possibility of translating the speckle vertically and perpendicularly to the flow. The particles' trajectories are tracked by digital video microscopy using the image projected by a microscope objective $(20 \mathrm{X}, \mathrm{NA}=0.5)$ and a tube lens (L2) onto a color CMOS camera. (b) A typical speckle pattern for optical manipulation as observed on the camera and (c) its 
normalized spatial autocorrelation function, which (d) permits us to characterize the average speckle grain size as the FHWM of the autocorrelation along the axes (solid lines).

\section{CONCLUSIONS}

In conclusion, we have shown an experimental setup based on the use of a multimode optical fiber to perform optical manipulation of microparticles in microfluidic flows based on static, time- or space-varying speckle fields as in Reference [34]. Our technique, beyond demonstrating that random potentials are a valid alternative to more regular potentials for the purpose of optical manipulation, offers some additional advantages to current optical manipulation techniques, such as intrinsic robustness to noise, and to optical aberrations from the optical systems and the sample. The experimental results obtained with this technique can be understood and interpreted in terms of numerical simulation based on Brownian dynamics [29,36-37].

\section{REFERENCES}

[1] Ashkin, A., "Acceleration and trapping of particles by radiation pressure," Phys. Rev. Lett. 24, 156-159 (1970).

[2] Ashkin, A. and Dziedzic, J.M., "Optical levitation by radiation pressure," Appl. Phys. Lett. 19, 283-285 (1971).

[3] Dholakia, K. and Čižmár, T., "Shaping the future of manipulation," Nature Photon. 5, 335-342 (2011).

[4] Padgett, M. and Bowman, R., "Tweezers with a twist," Nature Photon. 6, 343-348 (2011).

[5] Maragò, O.M., Jones, P.H., Gucciardi, P.G., Volpe, G. and Ferrari, A.C., "Optical trapping and manipulation of nanostructures," Nature Nanotech. 8, 807-819 (2013).

[6] Ashkin, A. and Dziedzic, J.M., "Optical trapping and manipulation of viruses and bacteria," Science 235, 1517 1520 (1987).

[7] McDonald, M.P., Spalding, G.C. and Dholakia, K., "Microfluidic sorting in an optical lattice," Nature 426, 421424 (2003).

[8] Jonáś, A. and Zemánek, P., "Light at work: the use of optical forces for particle manipulation, sorting and analysis," Electrophoresis 29, 4813-4851 (2008).

[9] Dufresne, E.R. and Grier, D.G., "Optical tweezers arrays and optical substrates created with diffractive optics," Rev. Sci. Instrumen. 69, 1974-1977 (1998).

[10]Reicherter, M., Haist, T., Wagemann, E.U. and Tiziani, H.J., "Optical particle trapping with computergenerated holograms written on a liquid-crystals display," Opt. Lett. 24, 608-610 (1999).

[11] Milne, G., Rhodes, D., McDonald, M. and Dholakia, K., "Fractionation of polydisperse colloid with acoustooptically generated potential energy landscapes," Opt. Lett. 32, 1144-1146 (2007).

[12] Volpe, G., Volpe, G., and Quidant, R., "Fractal plasmonics: subdiffraction focusing and broadband spectral response by a Sierpinski nanocarpet," Opt. Express 19, 3612-3618 (2011).

[13] Ricárdez-Vargas, I., Rodríguez-Montero, P., Ramos-García, R. and Volke-Sepúlveda, K., "Modulated optical sieve for sorting of polydisperse microparticles," Appl. Phys. Lett. 88, 121116 (2006).

[14]Čižmár, T., Šiler, M., Šerý, M., Zemánek, P., Garcés-Chávez, V. and Dholakia, K., "Optical sorting and detection of submicrometer objects in a motional standing wave," Phys. Rev. B 74, 035105 (2006).

[15] Albaladejo, S., Marqués, M.I., Scheffold, F. and Sáenz, J.J., "Giant enhanced diffusion of gold nanoparticles in optical vortex fields," Nano Lett. 9, 3527-3531 (2009).

[16]Demergis, V. and Florin, E.L., "High precision and continuous optical transport using a standing wave optical line trap," Opt. Express 19, 20833-20848 (2011).

[17] Šiler, M., Čižmár, T. and Zemánek, P., "Speed enhancement of multi-particle chain in a traveling standing wave," Appl. Phys. Lett. 100, 051103 (2012).

[18] Volpe, G., Quidant, R., Badenes, G., and Petrov, D., "Surface Plasmon Radiation Forces," Phys. Rev. Lett. 96, 238101 (2006).

[19] Applegate Jr., R.W., Squier, J., Vestad, T., Oakey, J., Marr, D.W.M., Bado, P., Dugan, M.A. and Said, A.A., "Microfluidic sorting system based on optical waveguide integration and diode laser bar trapping," Lab Chip $\mathbf{6}$, 422-426 (2006).

[20] Goodman, J.W., "Some fundamental properties of speckle," J. Opt. Soc. Am. 66, 11451150 (1976).

[21] Boiron, D., Mennerat-Robilliard, C., Fournier, J.M., Guidoni, L., Salomon, C. and Grynberg, G., "Trapping and cooling cesium atoms in a speckle field," Eur. Phys. J. D 7, 373-377 (1999). 
[22] Shvedov, V.G., Rode, A.V., Izdebskaya, Y.V., Leykam, D., Desyatnikov, A.S., Krolikowski, W. and Kivshar, Y.S. "Laser speckle field as a multiple particle trap," J. Opt. 12, 124003 (2010).

[23] Shvedov, V.G., Rode, A.V., Izdebskaya, Y.V., Desyatnikov, A.S., Krolikowski, W. and Kivshar, Y.S., "Selective trapping of multiple particles by volume speckle field," Opt. Express 18, 3137-3142 (2010).

[24] Staforelli, J.P., Brito, J.M.,Vera, E., Solano, P. and Lencina, A.A., "A clustered speckle approach to optical trapping," Opt. Commun. 283, 4722-4726 (2010).

[25]Hanes, R.D.L., Dalle-Ferrier, C., Schmiedeberg, M., Jenkins, M.C. and Egelhaaf, S.U., "Colloids in one dimensional random energy landscapes," Soft Matter 8, 2714-2723 (2012).

[26]Douglass, K.M. Sukhov, S. and Dogariu, A., "Superdiffusion in optically controlled active media," Nature Photon. 6, 834-837 (2012).

[27]Evers, F., Zunke, C., Hanes, R.D.L., Bewerunge, J., Ladadwa, I., Heuer, A. and Egelhaaf, S.U., "Particle dynamics in two-dimensional random-energy landscapes: Experiments and simulations," Phys. Rev. E 88, 022125 (2013).

[28]Evers, F., Hanes, R.D.L., Zunke, C., Capellmann, R.F., Bewerunge, J., Dalle-Ferrier, C., Jenkins, M.C., Ladadwa, I., Heuer, A., Castañeda-Priego, R. and Egelhaaf, S.U., "Colloids in light fields: Particle dynamics in random and periodic energy landscapes," Eur. Phys.J. Special Topics 222, 2995-3009 (2013).

[29] Volpe, G., Volpe, G. and Gigan, S. "Brownian Motion in a Speckle Light Field: Tunable Anomalous Diffusion and Selective Optical Manipulation," Sci. Rep. 4, 3936 (2014).

[30]Čižmár, T., Mazilu, M. and Dholakia, K., "In situ wavefront correction and its application to micromanipulation," Nature Photon. 4, 388-394 (2010).

[31]Bianchi, S. and Di Leonardo, R. "A multi-mode fiber probe for holographic micromanipulation and microscopy," Lab Chip 12, 635-639 (2012).

[32]Faucheux, L.P., Bourdieu, L.S., Kaplan, P.D. and Libchaber, A. J., "Optical Thermal Ratchets," Phys. Rev. Lett. 74, 1504-1507 (1995).

[33]Lee, S.H., Ladavac, K., Polin, M. and Grier, D.G., "Observation of flux reversal in a symmetric optical ratchet," Phys. Rev. Lett. 94, 110601 (2005).

[34] Volpe, G., Kurz, L., Callegari, A., Volpe, G. and Gigan, S. "Speckle Optical Tweezers: micromanipulation with random light fields," Opt. Express 22, 18159-18167 (2014).

[35] Crocker, J.C. and Grier, D.G. "Methods of Digital Video Microscopy for Colloidal Particles," J. Colloid. Interfac. Sci. 179, 298-310 (1996).

[36] Volpe, G. and Volpe, G., "Simulation of a Brownian particle in an optical trap," Am. J. Phys. 81, 224-230 (2013).

[37] Volpe, G., Gigan, S. and Volpe, G., "Simulation of the active Brownian motion of a microswimmer," Am. J. Phys. 82, 659-664 (2014). 